\title{
The search for social validation and the sexual behavior of people living with HIV in Rio de Janeiro, Brazil: Understanding the role of treatment optimism in context
}

\author{
Deanna Kerrigan $^{\mathrm{a}, *}$, Francisco I. Bastos ${ }^{\mathrm{b}}$, Monica Malta ${ }^{\mathrm{b}}$, \\ Claudia Carneiro-da-Cunha ${ }^{b}$, J.H. Pilotto ${ }^{b}$, Steffanie A. Strathdee ${ }^{c}$ \\ ${ }^{a}$ School of Public Health, Johns Hopkins, $615 \mathrm{~N}$ Wolfe St. E5523A, Baltimore, MD 21205, USA \\ ${ }^{\mathrm{b}}$ Oswaldo Cruz Foundation, Rio de Janeiro, Brazil \\ ${ }^{\mathrm{c}}$ University of California, San Diego, USA
}

Available online 28 November 2005

\begin{abstract}
The primary aim of this qualitative study was to explore the influence of HIV treatment optimism on the sexual behavior of people living with HIV/AIDS (PLWHA) receiving highly active anti-retroviral therapy (HAART) at public health clinics in Rio de Janeiro, Brazil. We also explored the psycho-social dynamics of participants' sexual relationships in order to understand more broadly how these factors influence the sexual behavior of PLWHA and how they shape HAARTrelated beliefs. Twenty-three semi-structured, in-depth interviews were conducted with three groups: heterosexual women, heterosexual men and men who have sex with men living with HIV who reported being sexually active in the last year and were currently receiving HAART. We found that the availability of HAART was conceptualized as a rationale for unsafe sex among a minority of study participants and that this was more common among men than among women. Specific examples of treatment optimism appeared to be employed as a means to avoid acknowledging how deeper psychosocial issues may have been influencing participant's sexual behavior. Most participants' sexual behavior appeared largely to be a product of their desire for social validation and linked to feelings of shame and denial, including but not limited to HIV. Participants from all three groups expressed a considerable amount of fear and/or anxiety regarding behaviors such as disclosure and condom use as a result of the unexplored conflict between implementing these behaviors and continuing with their strategies for social validation within the context of their sexual relationships. We conclude that short-term information, education and communication interventions surrounding treatment optimism, disclosure and condom use are appropriate and necessary; but that they are not sufficient to address the core challenges to unsafe sex among PLHWA. These deep-rooted psychosocial issues may be better addressed by longer-term individual and group-level opportunities for exploration and critical reflection regarding sense of self and its relationship to social solidarity among PLWHA.
\end{abstract}

(C) 2005 Elsevier Ltd. All rights reserved.

Keywords: HIV/AIDS; HAART; Sexual behavior; Treatment optimism; Brazil

\footnotetext{
${ }^{*}$ Corresponding author. Tel.: + 14106140075.

E-mail addresses: dkerriga@jhsph.edu (D. Kerrigan), chicao29@hotmail.com (F.I. Bastos),mmalta@jhsph.edu (M. Malta), caurids@hotmail.com (C. Carneiro-da-Cunha), pilotto@unisys.com.br (J.H. Pilotto), sstrathdee@ucsd.edu (S.A. Strathdee).
}

\section{Introduction}

HIV treatment optimism is a concept which has garnered a significant amount of attention in recent 
years, particularly since highly active anti-retroviral therapy (HAART) became available to people living with HIV in higher-income countries during the mid-to-late 1990s. The term generally refers to individual's decreased perceived need for protective sexual behaviors, such as condom use, as a result of their beliefs surrounding HAART. These beliefs commonly focus on the idea that people living with HIV are less infectious because they are taking HAART and/or that HIV infection is generally less of a threat to one's health because of the availability of HAART (Remien \& Smith, 2000). Numerous cross-sectional studies have been conducted in order to examine the relationship between these optimistic beliefs and condom use, mostly among men who have sex with men (MSM) in higher income countries. Findings from such studies have been mixed, with some studies documenting significant associations between treatment optimism and lower rates of condom use (Ostrow et al., 2002; Van de Ven, Kippax, Knox, Prestage, \& Crawford, 1999) and others not (Elford, Bolding, Maguire, \& Sherr, 2000; Van der Straten, Gomez, Saul, Quan, \& Padian, 2000). However, a recent meta-analysis based on data synthesized from over 25 crosssectional studies concluded that the prevalence of unsafe sexual behaviors was significantly elevated among individuals, regardless of their HIV serostatus, who possessed optimistic beliefs related to HAART (Crepaz, Hart, \& Marks, 2004).

Recently, two longitudinal studies seeking to understand potential causal relationships between increased sexual risk taking and HIV treatment optimism have been conducted. Huebner et al. found a significant association between optimistic beliefs regarding HAART and decreases in condom use over time. Additional analyses, however, demonstrated that optimism among the HIV-negative MSM study participants followed, rather than preceded, decreases in condom use (Huebner, Rebchook, \& Kegeles, 2004). Stolte et al. also found significantly increased odds of unsafe sex over time among HIV-negative MSM who perceived HIV as less of a threat due to HAART (Stolte, de Wit, van Eeden, Coutinho, \& Dukers, 2004; Stolte, Dukers, Geskus, Coutinho, \& de Wit, 2004) and concluded that optimism predicted increases in sexual risk behavior. The only longitudinal study among people living with HIV/AIDS (PLWHA) measuring HAART-related beliefs, published to date, was conducted among a small sample of MSM and found that perceptions of viral load were associated with increases in sexual risk behavior (Stolte, de Wit et al., 2004; Stolte, Dukers et al., 2004). Whether treatment optimism is utilized as a rationale for unprotected sex by some individuals, prior to or following unprotected sex, it is important to understand what types of psycho-social dynamics may lead some people to develop optimistic beliefs and/or utilize them as a justification for unsafe sex in either scenario. While limited research has been conducted on this issue, there are important indications that perceptions of self and identity, as well as community, are inextricably interwoven with beliefs surrounding HAART and that these dynamic belief patterns have an important influence on the health behaviors of PLWHA (Bartos \& McDonald, 2000).

The role of HAART-related beliefs on the sexual behavior of PLWHA or those at heightened risk for HIV infection from lower income countries is of critical importance as prevention efforts strive to slow the spread of the HIV epidemic globally. Brazil is one of the only middle to lower income countries to have thus far established universal access to HAART for all PLWHA Brazilian federal law, passed in 1996 mandates access to HAART for all PLWHA who are either clinically ill and/or meet specific clinical criteria related to CD4 count and viral load (BMOH, 2000a, 2000b). There are currently more than 660,000 PLWHA in Brazil (UNAIDS, 2004), of which approximately 150,000 are currently receiving HAART (Personal communication/BMOH, 2005). In turn, Brazil is a unique environment for research related to both treatment optimism as well as other contextual factors influencing the sexual behavior of PLWHA. To date, few studies have been conducted on these topics in Brazil. Hence, the primary aim of this qualitative study was to explore the potential role of HIV treatment optimism on the sexual behavior of PLWHA receiving HAART at public health clinics in Rio de Janeiro, Brazil. We also inquired into the psycho-social dynamics of participants' sexual relationships in order to more broadly understand how these factors may influence the sexual behavior of PLWHA as well as how they may shape HAART-related beliefs.

\section{Methods}

Thirty semi-structured, in-depth interviews were conducted among PLWHA in Rio de Janeiro, Brazil during the Fall of 2001. We purposively 
sampled 10 heterosexual women, 10 heterosexual men and 10 men who have sex with men who met the following selection criteria based on clinic records: diagnosed as HIV-positive and currently receiving clinical care and HAART from one of three designated public health clinics. Participants were considered heterosexual for the purposes of the study if they reported no other same sex partners in the last year. Male participants were considered MSM if they reported having at least one male sexual partner in the last year. Of the 30 individuals interviewed, 23 reported having at least one sexual partner in the last year (seven women, eight heterosexual men, and eight men who have sex with men) and are included in the analysis presented herein.

Study clinics were selected based on their prior participation in HIV-related research and the fact that they were all large public health clinics who generally serve lower income individuals and all offer free HIV-related clinical care and HIV medications to a diverse array of PLWHA. On average each of the participating clinics were serving approximately 1000 PLWHA on an ongoing basis at the time of the study.

A physician or social worker from the clinic assisted the study team by asking patients who appeared to meet the study selection criteria whether they were interested in hearing more about a study being conducted among PLWHA receiving HAART. Potential participants were brought to a private room in the clinic. Interviewers first confirmed via a brief screening tool whether participants met selection criteria and then interviewers explained the study aims, methods, risks and benefits of participation to those eligible individuals. After hearing about the study, participants signed an informed consent form if they agreed to participate. The interview lasted approximately $1 \mathrm{~h}$. All participants were offered \$US7 for their participation in the study. The study was approved by the Committees on Human Research at both of the research institutions involved in the study, the Johns Hopkins Bloomberg School of Public Health in Baltimore, Maryland in the United States and the Oswaldo Cruz Foundation (FIOCRUZ) in Rio de Janeiro, Brazil, as well as the Brazilian National Ethics Committee (CONEP).

Key topics explored through the in-depth interview process included the structure and dynamics of participants' daily lives; relationships with family, friends and sexual partners; knowledge, attitudes, beliefs and practices related to HAART, and their thoughts on the potential role of access to HAART on the sexual behavior of PLWHA. All in-depth interviews were conducted in Portuguese. They were audio-taped and later transcribed, keeping the texts in Portuguese. Transcriptions were first read several times in their entirety. A list of domains of interest and codes within each domain were developed based on the reading of transcriptions and the content of interview field guides. Primary analytical domains and associated codes emphasized in this analysis include: 'living with HIV' (e.g. relationship to diagnosis, experiences with HAART, and the role of HAART on safe sex) and 'romantic relationships' (e.g. interpersonal communication, social exchange and sexual behavior). Textual data was coded using the qualitative software analysis program, Atlas.ti ${ }^{\odot}$. All codes were run and synthesized within population groups. Salient themes resulting from the synthesis of code output were then written up as memos and compared across population groups. Quotes selected were translated for the purpose of presentation in the manuscript. The narrative for the manuscript was then developed after re-reading all interviews in order to situate themes and quotes in context.

\section{Results}

Socio-demographic and behavioral characteristics of the sample

Study participants were generally middle aged, with low levels of formal education and income. The median age was similar across the three groups interviewed: $37.0(26-45)$ among heterosexual women, 38.5 (26-52) among heterosexual men and 40.0 (22-49) among men who have sex with men. In terms of education, $35 \%(8 / 23)$ of participants had some primary schooling, 26\% (6/23) finished primary school, $22 \%(5 / 23)$ had some secondary school and $17 \%(4 / 23)$ had finished secondary school. Only one female participant had attended secondary school. Many participants were not employed at the time of the study. However, 3/7 women, $3 / 8$ heterosexual men and $4 / 8$ men who have sex with men reported having some form of paid employment. The median monthly income was similar among study groups: \$US89 (0-178) among women, SUS80 (0-356) among heterosexual men and SUS89 (0-244) among men who have sex with men. 
The median number of sexual partners in the last year was $1.0(1-10)$ among female participants, with all seven female participants reporting being in steady relationships. None of the women reported being married. All female participants had children; median 2.0 (1-7). The median number of sexual partners in the last year was also $1.0(1-10)$ among heterosexual men. Five of the eight heterosexual men reported currently being in a steady, ongoing partnership and two reported being married. Six out of eight heterosexual men reported having children; median $1.0(0-3)$. The median number of sexual partners in the last year was a bit higher among the MSM interviewed; 3.0 (1-10). Two of the eight MSM reported currently being in a steady partnership and none reported being married. One MSM reported having a child. There was one heterosexual man and one MSM, respectively; who stated that they had too many partners in the last year to remember the exact number and hence they were excluded from the median calculated for each group. There was a wide range among participants in terms of the length of time since being diagnosed with HIV. The median year of diagnosis with HIV was 1999 (1992-2000) for heterosexual women; 1997 (1990-2000) for heterosexual men; and 1997 (1992-2000) for men who have sex with men.

\section{Heterosexual women}

None of the women living with HIV interviewed connected their current or recent sexual behavior to optimistic beliefs surrounding HAART, nor did they feel that other PLWHA associated HAART with decreased perceptions of vulnerability and in turn increased risk behavior. Instead, many of the women interviewed emphasized the challenges they faced taking and striving to adhere to HAART and in turn found it difficult to envision how this experience could generate optimistic beliefs of any kind. For example, when asked if she thought PLWHA may be engaging in increased risk behavior due to HAART, one female participant stated:

I don't think so... those that take these medications know that it is extremely difficult to take them at the right time... we suffer a lot...so it would be hard for someone to think that way (37 years old, diagnosed with HIV in 1996).

Additionally, several female participants, when asked about the potential for treatment optimism, suggested that most PLWHA "take care of themselves" by using condoms and that those who do not are not likely to be motivated by access to HAART, but rather by the intention to infect others. This sentiment is expressed in the following quote:

You have people who don't use them [condoms], but it's not because of the medicines, it's because they actually want to pass it [HIV] to others and it's really intentional. No one does this thinking that there is no problem because of treatment; those who are on medication know that this is not an easy life. So it's either craziness or assassination (27 years old, diagnosed with HIV in 2000).

However, when asked to discuss their own sexual behavior and whether they used condoms with their current partners, female participants discussed several key challenges to safe sex, beyond that of treatment optimism or purposefully infecting others. The primary issue inhibiting safe sex in their relationships, according to several female participants, was fear.

Two of the seven women interviewed had not disclosed their HIV-status to their current regular sexual partner and reported difficulties negotiating condom use which they perceived to be associated with their inability to disclosure. Their fears related to disclosure tended to center on the potential for negative partner reactions including rejection, abandonment and violence. Such concerns are narrated below by a female participant who had lived through the struggle to hide her HIV status several times before:

I have to keep on hiding, because maybe he could even try to hurt me. If he knew he would definitely leave me. I use condoms sometimes...Could he hit me, could he kill me? I just can't tell him...this HIV has been the end of three marriages of mine (26 years old, diagnosed with HIV in 1997).

Both of the two women interviewed who had not disclosed their HIV status to their partner stated that convincing their partner of the need to use condoms without giving away their HIV status was very difficult. The following quote gives voice to what was described by these two female participants as their partner's typical reaction to the idea of using condoms: 
'You are a healthy woman, you don't have any disease... a dignified woman like yourself and you keep demanding condoms... why?' (38 years old, diagnosed with HIV in 1996).

Additionally, these two participants suggested that not having disclosed their HIV status at the beginning of the relationship made them feel that it was almost impossible to now introduce the subject because their partner would be upset about having been "doubly deceived".

The other five women interviewed were in ongoing relationships where their partner knew of their HIV status. In only one of these cases were both partners known to be infected and in that case the participant reported always using condoms with that partner. Despite their partners' knowing about their HIV status and their partner being either HIVnegative or of unknown status, negotiating condom use was still reported as a formidable challenge by all four of these other female participants. The reasons given by the women interviewed who had disclosed their HIV status to their partner but were not using condoms (3/4) were almost identical to those reported by the two women who had not disclosed their HIV status. In both cases the women stated that they were not practicing protective sex because their male partners did not want to use condoms due to a perceived sense of decreased sexual pleasure when using condoms and/or a desire to have more children.

While none of the women interviewed expressed their own desire to have more children due to their HIV status, the issue of their male partners desire to have children was a theme that emerged as an important barrier to safe sex among several female participants. One participant cited the number of HIV-positive pregnant women that she knew as "evidence" of the amount of unsafe sex that was occurring among PLWHA. Another participant who had not disclosed her HIV status to her partner, but who had told him how she had been sterilized, reported that even that did not stop her partner from pressuring her to have children with him. As the following quote conveys the desire to have children among male partners may reflect the internalization of deep-rooted social norms regarding fertility and masculinity:

I told him how my tubes are tied, but he said that with time these procedures could be reversed...that over time a woman could become pregnant again... he has made so many plans for us to become pregnant, he even has names picked out; he says that when the relationship between a man and a woman is good, then one must have a child, so that it stays that way and gets even better. But I know I cannot have another child, and even if I could, I don't want to (38 years old, diagnosed with HIV in 1996).

This participant was clear in her interview that she did not want children more children and reported wanting to use condoms in order to avoid potentially infecting her HIV-negative partner. However, from her perspective, insisting on condom use had the potential to break up her relationship and it was clear from the interview that she was simply not ready to live with that consequence.

\section{Heterosexual men}

When asked if and how being on HAART may be impacting the sexual behavior of PLWHA, most heterosexual men interviewed agreed that it did not have a significant influence. Many of the heterosexual men interviewed suggested that most people living with HIV worked hard to protect themselves and others. However, several participants within this group also felt that there was an important amount of unsafe sex occurring among PLWHA. Similar to reports made by heterosexual women, common reasons cited by heterosexual men regarding why other PLWHA did not use condoms, involved some combination of denial, anger, and lack of concern for others, as reflected in the following quotes.

No, I don't think that anyone thinks that way about treatment because we know that it doesn't cure; if there were a vaccine that could cure us, then one could think that way. But since there isn't, it is just a lack of intelligence to think that way (37 years old, diagnosed with HIV in 1997).

No, that's a person [someone who does not use condoms] who is having difficulty accepting [their HIV status]... it's a selfish escape (40 years old, diagnosed with HIV in 1992).

When reporting on the factors which shape their own sexual behavior, however, the issues cited above, such as denial and anger, suggested to be key factors related to unsafe sex among other PLWHA, were again not emphasized among heterosexual men. Instead, several heterosexual male participants described their general frustration 
with using condoms because of problems sustaining an erection and/or enjoying sex, as well as in relation to their desire to have children. Unprotected sex was reported among both participants whose partners were positive $(1 / 2)$ and those who were negative $(2 / 3)$ or of unknown HIV status $(2 / 3)$. There was no apparent indication in the eight interviews conducted among heterosexual men that disclosing one's HIV status was associated with increased condom use. Instead of emphasizing fear of the loss of their relationship, the heterosexual men interviewed who did not disclose their status (3/ 8), reported not doing so because they did not want to be labeled as HIV-positive or treated differently due to HIV, even when they suspected their partner would treat them with compassion if they disclosed.

While being on HAART was not reported as having a direct relationship to the sexual behavior of any of the heterosexual men interviewed or among PLWHA in general, indirect examples of treatment optimism found their way into several descriptions of why heterosexual men did not always use condoms. Below we present a few scenarios in which HAART-related beliefs were indirectly linked to or gave meaning to the sexual behavior of two of the heterosexual men we interviewed, both of whom had regular female partners who were also known to be infected with HIV.

The fact that your viral load is zero doesn't mean that you cannot transmit the virus to someone. I believe that possibility does exist, it never happened to me, but it does exist...just because your viral load is zero doesn't mean you are cured.

Yet when asked whether he and his current regular partner who is also HIV-positive were using condoms, this same participant went on to say:

Not always, we don't always manage to use them... sometimes... we think about condoms in order to not have more children, but the idea of the disease [HIV] does not affect us... if we are going to get worse because we don't use condoms... we don't think about that...we receive information from our doctors... they say all is the same, everything is $\mathrm{OK}$, so we are not affected (26 years old, diagnosed with HIV in 1999).

It was clear in this interview that the participant "understood" the technical definitions of viral load and reinfection; but periodically hearing his doctor say that he and his wife were "OK" was interpreted as a sign that condoms were not imperative. He also reported that condoms were a barrier to the intimacy that he and his wife were trying to reestablish after having lived through the difficult news that they were both infected with HIV.

While the participant above discussed the need for condoms as a means to avoid having more children rather than avoiding reinfection, another heterosexual male participant, whose wife was also infected with HIV, discussed his desire to have more children and how being on HAART has influenced their thinking regarding protective sex:

If my viral load was undetectable one day and my wife wanted to think about this possibility [having more children], and the doctor said we were both OK...then perhaps. For example, I heard about a hospital nearby where they did a study and out of 100 patients who were pregnant...95\% of their children were born without the virus. I don't know if that study was for real, but this type of thing makes people who have HIV and want to have children relax, especially if they don't already have children (35 years old, diagnosed with HIV in 1998).

A few of the heterosexual men interviewed also suggested that other men they knew, who were not known to have HIV, may also be influenced by access to HAART. The first quote presented below is from a heterosexual male participant reflecting on how access to treatment may influence the general population as well as how it influenced his own thinking and behavior prior to receiving his diagnosis.

It's the same story as with cigarettes. My grandmother smoked her whole life, she died when she was 70, and she smoked until the end. So people say, so and so has been taking medication for 15 years, he doesn't even look like he has it [HIV]. So this facilitates the person to continue having unsafe sex... why? Because so and so is fine, so if one day I get HIV, I'll be fine too. I myself have even thought this way at times... I thought one day if I get sick, I know there are medicines out there. I will be sincere with you...I thought this a few times when I had gone out and not used a condom... I thought this to myself...it was my unconscious attempt to soothe myself in this way: 'if it happens, there are 
medicines' (35 years old, diagnosed with HIV in 1998).

A few other heterosexual male participants also suggested that HIV-negative men they knew may be becoming using treatment as a rationale for engaging in unsafe sex. For example, one participant described how some of his male friends had developed optimistic beliefs related to HAART based on seeing him do well on these medications:

I have friends who all go out without condoms. They think that they can just go get some medicine like me and everything will be OK. I tell them no. I even wanted to give them some educational materials, but they said, 'what for'? I tried to tell them it's not like that, it's not a pretty picture [having HIV], but they don't want to listen (35 years old, diagnosed with HIV in 2000).

\section{Men who have sex with men}

The idea that most PLWHA generally "take care of" and protect themselves and others was articulated by many of the MSM interviewed. However, similar to the heterosexual women and men interviewed, several MSM acknowledged that they know of a considerable number of PLWHA who do not protect themselves. When asked if being on HAART may influence the sexual behavior of PLWHA, some MSM said this was a possibility, but indicated that there were also other critical factors influencing the sexual behavior of PLWHA that they knew. The issues of purposefully infecting others and lack of self-acceptance came up again among many of the MSM interviewed in response to interviewers probing on what these other factors might be. These themes are reflected in the quote below, as expressed by several MSM participants, when asked about the possibility of access to treatment leading PLWHA to engage in increased sexual risk behavior.

Give someone HIV thinking about the fact that there is treatment out there? No... There are people that I know that simply pass it intentionally. They lack self love and love for the person they are with (45 years old, diagnosed with HIV in 1994).

Most of the specific examples of treatment optimism described by MSM were in regard to other people they know, both living with HIV and not living with HIV, rather than about themselves. For example, in reference to a fellow patient at the clinic he attends, one of the MSM interviewed stated:

When I began treatment here I heard a conversation of another patient. The next time I saw him he was covered in a rash. I asked him what was going on and he said, 'I have AIDS. I was an HIV carrier and I was having sex with my wife without condoms'. I said: You're crazy man. Now I don't see him here anymore. He was thinking that if he didn't have a high viral load he was not going to pass it [HIV] on (36 years old, living with HIV since 1999).

Talking about people in general, not specifically about other PLWHA, another MSM interviewed discussed the possibility of treatment optimism:

It depends, some protect themselves and some don't. I know people who don't use condoms. They think because of the cocktail they are not at risk anymore. But no one is talking about the side effects of these medicines. I think there should be more information out there. They're working, this is important for people to know, but not for everyone (32 years old, living with HIV since 1996).

Similar to both heterosexual women and men, the discussion of what influences safe sex shifted considerably when the MSM interviewed discussed their personal lives and sexual relationships. The issue of disclosure was discussed at length by MSM participants. In the case of the eight MSM in our sample, all of them reported that disclosing their HIV status was an extremely challenging issue. Most of the MSM stated that is was difficult to disclose to new or casual partners because it was not known how they would respond. Six of the eight MSM reported only casual partners in the last year and none reported disclosing their status to these partners. However, several of these participants stated that they generally used condoms with new partners, even if they were not ready to disclose their status to them due to fear of negative reactions. As one MSM participant stated:

If I meet someone, they would never know [has HIV]. I just chat them up normally, we hang out together... and if we have sex, I just use a condom (22 years old, living with HIV since 1999).

However, a few of the MSM participants interviewed reported that not being able to disclose their 
HIV status did impact their ability to negotiate safe sex with some casual sexual partners who were otherwise not interested in using condoms, as described below:

Sometimes they accept using condoms, but sometimes they don't. You can't force someone. If I was about to have sex with someone and I said I have HIV, I would be risking getting killed in that moment... they could really do this (47 years old, diagnosed with HIV in 1999).

In the course of the interviews with MSM, it became clear that struggles with disclosure and condom use were not limited to new partners, but also within the context of longer-term relationships. Two of the eight MSM interviewed had regular sexual partners. One of the two had disclosed to their steady partner who was also infected with HIV. Both reported using condoms. As is conveyed in the following quote, disclosure does not necessarily need to be a prerequisite for to condom use, even in the context of ongoing relationships:

I am getting closer to him, so I want to wait. I'm going to give it a little more time. For now it's just us and he hasn't become suspicious. We use condoms, just not with oral sex. If it's a more serious relationship, then that's different. In some time he will become suspicious, but for now we just started, it's only been a few months. But in a little while I am going to have to open up to him (36 years old, diagnosed with HIV in 1998).

The idea that there had been a change in participant's sexual behavior, for example having fewer sexual partners and using condoms more consistently, as a result of being diagnosed with HIV was a theme which was mentioned among many male participants, but was particularly salient among those MSM interviewed. The following quote describes this shift in thinking which was expressed by several MSM participants:

I am more conscious now, more mature. I am not going to say it was good that I got HIV because it is not good for anyone, but I have definitely matured a lot. It has changed me. It's as if I have another head now (36 years old, diagnosed with HIV in 1998).

Interestingly, the last two quotes presented above are from the same participant. It is important to point out that based on this participant's interview, there would appear to be a connection between accepting and learning from one's HIV diagnosis and protective sexual behavior, as the participant reports using condoms with his current partner. However, even given the reported evolution that has taken place since his diagnosis, the participant still finds it difficult to disclose his HIV status, even in the context of a regular partnership, indicating just how challenging becoming completely vulnerable to another can be.

\section{Discussion}

Findings from this qualitative study of HIVpositive persons receiving HAART in Brazil suggest that optimistic beliefs surrounding treatment are influencing the sexual behavior of a minority of study participants. Personal accounts of how perceptions of viral load and/or infectivity shaped HIV-positive participant's sexual behavior were described by a few heterosexual men only. A few heterosexual men and MSM, respectively, also reported that perceptions of viral load and infectivity influenced the sexual behavior of other PLWHA that they knew of or had interacted with. Some heterosexual men and MSM reported that access to treatment was a factor shaping the sexual behavior of a minority of HIV-negative individuals that they know, due to the perception that HIV is now less of a serious issue because of the availability of HAART. While qualitative in nature, our study suggests that HIV treatment optimism is occurring among a small percent of PLWHA and/or other HIV-negative individuals in the Brazilian context, a finding which is consistent with most other quantitative studies conducted to date (International Collaboration of HIV Optimism, 2003). It is important to note, that most of our study participants reported being satisfied with their current clinical care and receiving a significant amount of information regarding HAART from their providers. As access to HIV treatment is scaled up in other lower and middle income countries, it will be important to monitor how provider relationships and the provision of information regarding HAART correlates with levels of treatment optimism in other settings.

Our findings also suggest that there is a distinction regarding the salience of treatment optimism by gender; whereas none of the female participants from our study reported any personal sense of increased optimism related to HAART, nor did they 
report knowing of others who may have internalized such beliefs. A few previous studies have demonstrated gender differences in uptake of HAART among PLWHA, with significantly lower uptake documented among women (Ezzy, Bartos, de Visser, \& Rosenthal, 1998; Misener \& Sowell, 1998). Additionally, others have documented gender differences in attitudes regarding the efficacy of HAART among PLWHA (McDonald, Bartos, \& Rosenthal, 2001), with women being significantly less optimistic than men regarding the potential for positive health outcomes associated with HAART. However, we were unable to find any studies in the peer-review literature to date which have documented gender differences in treatment optimism as it has been conceptualized in this article, e.g. decreased need for protective sexual behavior due to the availability of HAART. Hence, exploring gender differences related to the relevance of treatment optimism across distinct social contexts will be an important line of inquiry for future research.

The personal accounts of HIV treatment optimism shared by a few heterosexual male study participants suggest that optimism is employed as a rationale for sexual risk behavior and is the product of deeper psycho-social dynamics. This finding was illuminated during study interviews as participants were in one moment able to clearly articulate in biomedical terms the potential for HIV transmission and reinfection despite lower viral loads, and in another moment shortly thereafter state that condoms were not imperative for them because they were told by care providers that they were 'OK' or because of the existence of new therapies for maternal child transmission. Interview exchanges regarding beliefs about treatment and the need for condoms were interwoven with expressions of participants' desire to reestablish intimacy with their partner and/or their desire for children. In the end, it appears that for some participants the desire for social connectedness and/or social validation simply outweighs biomedical information received regarding HAART, suggesting the priority placed on protecting the 'social self', rather than the physical body. Prior research conducted with women living with HIV in Brazil related to protected sex in the context of fertility decisionmaking also revealed that a heavier emphasis is often placed on the perceived 'social risks' associated with HIV-related health behaviors rather than biomedical risks (Maksud, 2002; Ventura, 2002). Broadly, these findings indicate the need for further longitudinal research regarding the temporal relationship between treatment optimism and increased sexual risk behavior which contemplates the role of other psycho-social factors which may predict and/or interact with optimism as it relates to the sexual behavior of PLWHA or those at heightened risk for infection.

In addition to HIV treatment optimism, there were several common themes that emerged from all three population groups regarding those factors considered to be important influences on the sexual behavior of PLWHA. While many participants suggested that PLWHA generally 'do a good job' of protecting themselves and others, there were also a number of participants who believed that some PLWHA who engaged in unsafe sex were intentionally trying to infect others. Reasons offered for why a given person living with HIV would purposely engage in such behavior were also fairly consistent across all three groups and involved a combination of denial, avoidance and anger about their HIV status as well as a general lack of self-acceptance which translated into a lack of compassion for others. However, when the conversations shifted to participant's personal lives and relationships there was very little acknowledgement of or self-reflection regarding the role of denial, anger or lack of selflove and compassion on their own sexual behavior. These findings suggest the challenge of conducting research on the issue of internalized stigma and shame among PLWHA. Clinical psychologists have suggested that by its very definition shame seeks to cover that which one perceives to be undesirable and employs behavioral strategies such as pleasure seeking, as documented among many of the men from this study, and compliance, as documented among several of the women in this study, in order to escape the anxiety and discomfort associated it (Brach, 2003). In turn, the development and utilization of innovative research and intervention methodologies which seek to make conscious that which is generally unconscious will be needed in order to further understand and address the role of shame in the sexual health of PLWHA.

Strategies for avoiding and covering the discomfort associated with shame such as seeking social approval and/or seeking sexual pleasure provided the contextual backdrop to almost all of the discussions with participants regarding the challenges to disclosing one's serostatus and/or using condoms. Fears of being discredited and abandoned and in turn strategies of compliance with partner 
desires and demands were particularly salient among female participants as well as MSM. Desires to feel socially validated by having more children were more common among heterosexual men as were strategies of pleasure-seeking through sexual experience. It is also important to note that strong desires for children among HIV-positive men have been documented by prior research conducted among PLWHA in Brazil (Paiva, Filipe, Santos, Lima, \& Segurado, 2003) and the United States (Chen, Philips, Kanouse, Collins, \& Miu, 2001), indicating the importance of future inquiry into this issue. More generally, the fears and desires expressed by study participants suggest that participants tend to define themselves based upon their interactions with and recognition from others. These findings, as well as that of prior research, suggest that sense of self has an important relationship with both the mental and sexual health of PLWHA (Bartos \& McDonald, 2000; Green \& Sobo, 2000). While the ways in which a given individual may seek to negotiate the social risks of integrating HIV into one's sense of self may vary per gender, sexual orientation and/or socio-cultural context, the overall desire for social validation is universal and in turn should be considered as an important component of conceptual frameworks related to future intervention research regarding HIV and health behaviors.

Our findings do not suggest that there is necessarily a linear relationship between disclosure of HIV status and safer sexual behaviors among PLWHA, nor do they suggest that disclosure should be considered a necessary prerequisite to practicing safer sex. Instead, a more nuanced and dynamic relationship between disclosure and safer sex appears to be occurring among many study participants, situated within the context of other factors such as attitudes towards their own HIV diagnosis, partner relationship dynamics and perceptions of risk related to their partner's HIV status. The importance of understanding the psycho-social context of disclosure of HIV with regard to its relationship with protective sexual behavior, rather than focusing on the behavior of disclosure only, has been emphasized in prior research conducted among PLWHA (Gorbach et al., 2004; Marks \& Crepaz, 2001). Additionally, a recent review of the relationship between disclosure of HIV and safer sex suggests the importance of more in-depth, contextual research on this topic, given the conflicting results documented to date regarding the potential association between the two phenomenons (Simoni \& Pantalone, 2004).

We would like to acknowledge potential limitations to our study including our sample size and in turn our ability to generalize study results to a broader audience of PLWHA. Additionally, we spoke to study participants at one point in time and were unable to follow the development and interconnection of the constructs examined over time. With these limitations in mind, our findings indicate that psycho-social issues including fear, shame and anxiety related to, but not limited to, living with HIV, merit further research among PLWHA. Additionally, our data suggest that until these issues are brought into awareness, it will be difficult to sustain behavioral change related to sexual health among PLWHA via informational and motivational interventions alone. While health education surrounding treatment optimism, disclosure and condom use are appropriate and necessary; our findings suggest that they are not sufficient to address the core challenges to unsafe sex among PLWHA. These underlying psycho-social issues may be better addressed by longer-term individual and group-level opportunities for exploration, critical reflection and social solidarity among PLWHA. We must emphasize that we do not suggest that participant's fears and anxieties are limited to their experience with HIV or that the search for social validation is unique to PLWHA. Fear, shame and anxiety are clearly set within a larger social context of material and social inequality and alienation experienced by many. However, developing an awareness of how the experience of our relationships and surroundings shapes individual and collective agency is an important first step in any larger social movement (Freire, 2003), including one which aspires to break the circle of shame and blame surrounding HIV.

\section{Acknowledgement}

The authors would like to thank all of the study participants who gave generously of their time and of themselves to benefit others. We would like to thank all of the clinic staff at the three public health centers, the Hospital Geral de Nova Iguacu, the Ambulatorio da Providencia and the Instituto de Pesquisas Evandro Chagas, where we conducted this research including Rosa Maria Pinheiro Rezende, Monica Fortuna, and Maria Ines Linhares de Carvalho. We would also like to thank our 
funding sources including the Johns Hopkins University Center for AIDS Research (CFAR), Grant P30 AI42855, and the National Institute of Mental Health, Grant K01 MH3491.

\section{References}

Bartos, M., \& McDonald, K. (2000). HIV as identity, experience or career. AIDS Care, 12(3), 299-306.

Brach, T. (2003). Radical acceptance: Embracing your life with the heart of a Buddha. New York: Bantam Dell.

Brazilian Ministry of Health (BMOH) (2000a). Brazilian legislation on STD and AIDS. Brasilia: Brazilian Ministry of Health (in Portuguese).

Brazilian Ministry of Health (BMOH) (2000b). Recommendations for antiretroviral therapy in HIV-infected adults and adolescents. Available online (in Portuguese).

Chen, J. L., Philips, K. A., Kanouse, D. E., Collins, R. L., \& Miu, A. (2001). Fertility desires and intentions of HIVpositive men and women. Family Planning Perspectives, 33(4), $144-152 \& 165$.

Crepaz, N., Hart, T. A., \& Marks, G. (2004). Highly active antiretroviral therapy and sexual risk behavior: A metaanalytic review. Journal of American Medical Association, 292(2), 224-236.

Elford, J., Bolding, G., Maguire, M., \& Sherr, L. (2000). Combination therapies for HIV and sexual risk behavior among gay men. Journal of Acquired Immune Deficiency Syndromes, 23(3), 266-271.

Ezzy, D. M., Bartos, M. R., de Visser, R. O., \& Rosenthal, D. A. (1998). Antiretroviral uptake in Australia: Medical, attitudinal and cultural correlates. International Journal of STD and AIDS, 9(10), 579-586.

Freire, P. (2003). Pedagogy of the Oppressed. New York, London: Continuum.

Gorbach, P. M., Galea, J. T., Amani, B., Shin, A., Celum, C., Kerndt, O., \& Golden, M. R. (2004). Don't ask, don't tell: Patterns of HIV disclosure among HIV positive men who have sex with men with recent STI practicing high risk behaviour in Los Angles and Seattle. Sex Transmitted Infections, 80(6), 512-517.

Green, G., \& Sobo, E. (2000). The endangered self: Managing the social risks of HIV. London, New York: Routlegde.

Huebner, D. M., Rebchook, G. M., \& Kegeles, S. M. (2004). A longitudinal study of the association between treatment optimism and sexual risk behavior in young adult gay and bisexual men. Journal of Acquired Immune Deficiency Syndromes, 37(4), 1514-1519.

International Collaboration on HIV Optimism. (2003). HIV treatments optimism among gay men: An international perspective. Journal of Acquired Immune Deficiency Syndromes, 32, 545-550.

Maksud, I. (2002). Casais com sorologias distintas para o HIV: Questões inicias para debate. In I. Maksud, et al. (orgs).
Conjugalidade e AIDS: A questão da sorodiscordância e os serviços de saúde. Rio de Janeiro, Brazil: ABIA.

Marks, G., \& Crepaz, N. (2001). HIV-positive men's sexual practices in the context of self-disclosure of HIV status. Journal of Acquired Immune Deficiency Syndromes, 27(1), 79-85.

McDonald, K., Bartos, M., \& Rosenthal, D. (2001). Australian Women Living with HIV/AIDS are more skeptical than men about antiretroviral treatment. AIDS Care, 13(1), 15-26.

Misener, T. R., \& Sowell, R. L. (1998). HIV-infected women's decisions to take antiretrovirals. Western Journal of Nursing Research, 20(4), 431-447.

Ostrow, D. E., Fox, K. J., Chmiel, J. S., Silvestre, A., Visscher, B. R., Vanable, P. A., et al. (2002). Attitudes towards highly active antiretroviral therapy are associated with sexual risk taking among HIV-infected and uninfected homosexual men. AIDS, 16(5), 775-780.

Paiva, V., Filipe, E. V., Santos, N., Lima, T. N., \& Segurado, A. (2003). The right to love: The desire for parenthood among men living with HIV. Reprod Health Matters, 11(22), 91-100.

Personal communication (2005). Communication with Cristina Possas, director of? Brazilian Ministry of Health, Brasilia.

Remien, R. H., \& Smith, R. A. (2000). HIV prevention in the era of HAART: Implications for providers. AIDS Reader, 10(4), 247-251.

Simoni, J., \& Pantalone, D. (2004). Secrets and safety in the age of AIDS: Does HIV disclosure lead to safer sex? Topics in HIV Medicine, 12(4), 109-118.

Stolte, I. G., de Wit, J. B., van Eeden, A., Coutinho, R. A., \& Dukers, N. H. (2004). Perceived viral load, but not actual HIV-1-RNA load, is associated with sexual risk behaviour among HIV-infected homosexual men. AIDS, 18(14), 1943-1949.

Stolte, I. G., Dukers, N. H., Geskus, R. B., Coutinho, R. A., \& de Wit, J. B. (2004). Homosexual men change to risky sex when perceiving less threat of HIV/AIDS since availability of highly active antiretroviral therapy: A longitudinal study. AIDS, 18(2), 303-309.

United Nations Programme on HIV/AIDS (UNAIDS) (2004). Epidemiological fact sheets on HIV/AIDS and sexually transmitted diseases: Brazil. 2004 Update. Geneva: World Health Organization.

Van de Ven, P., Kippax, S., Knox, S., Prestage, G., \& Crawford, J. (1999). HIV treatments optimism and sexual behaviour among gay men in Sydney and Melbourne. AIDS, 13(16), 2289-2294.

Van der Straten, A., Gomez, C. A., Saul, J., Quan, J., \& Padian, N. (2000). Sexual risk behaviors among heterosexual HIV serodiscordant couples in the era of post-exposure prevention and viral suppressive therapy. AIDS, 14(4), F47-F54.

Ventura, M. (2002). Conjugalidade e AIDS: A perspectiva dos direitos humanos. In I. Maksud, et al. (orgs). Conjugalidade e AIDS: A questão da sorodiscordância e os serviços de saúde. Rio de Janeiro, Brazil: ABIA. 